\section{High-Resolution Impedance Inversion}

Carlos Cunha*, Andrea Damasceno, Anderson Pimentel, Leonardo Teixeira, Nathalia Cruz, Tatiana de Oliv eira, PETROBRAS.

Copyright 2019, SBGf - Sociedade Brasileira de Geofísica

This paper was prepared for presentation during the $16^{\text {th }}$ International Congress of the Brazilian Geophysical Society held in Rio de Janeiro, Brazil, 19-22 August 2019.

Contents of this paper were reviewed by the Technical Committee of the $16^{\text {th }}$ International Congress of the Brazilian Geophysical Society and do not necessarily International congress of the Brazlan Geophysical society and do not necessar ily represent any position of the SBGF, its officers or members. Electronic reproduction or storage of any part of this paper for commercial purposes without the written consent of the Brazilian Geophysical Society is prohibited.

\section{Abstract}

We present a new methodology, and preliminary results attained by incorporating bandwidth expansion techniques in acoustic and elastic impedance inversions. We discuss the required adaptations in the sparse-spike inversion workflow, and present the advantages of this approach when compared with conventional inv ersion results.

\section{Introduction}

Many internal processes were dev eloped along the last two decades in Petrobras with the goal to improve seismic resolutions. Applications of these processes were limited to qualitative analysis in which faciologic, lithologic and fluid characterizations are correlated to relative physical quantities. These applications include internal publications regarding the characterization of carbonate reserv oirs of the pre-salt in the Santos and Campos Basins and 4-D analy sis of turbidities in the Campos Basin, facies mapping of the pre-salt reserv oirs in Campos basin (Cunha e outros, 2013). On the other hand, adv ances on seismic acquisition and processing improved the reliability of the seismic amplitude, thereby the quantitative interpretation of turbidite and pre-salt reservoirs based on impedance volumes became more trustworthy and integrated with static and dy namic data (Teixeira et al., 2017). However, impedance volumes are restricted to the bandwidth of the input data and, therefore, have not benefited from the bandwidth expansion processes.

Seismic quantification of rock properties relies on the combination of rock phy sics and seismic inv ersion (Av seth et al., 2005; Vernik, 2016). Rock physics links the petrophysical and elastic properties. It evaluates how saturating fluid, porosity, clay content, pore shape, mineralogy, pressure affect the seismic signal. Sparsespike seismic inv ersion enables the spatial extrapolation of well-derived elastic properties by inverting the migrated traces into elastic properties (Latimer, 2011). The incorporation of high-frequency bandwidth in seismic inversion significantly improves the reservoir characterization, with quantitative characterization of the depositional system geometry, and rock properties with resolution more compatible with the scales inv olv ed in flow simulators.

\section{Iterative Deconvolution and High-Resolution Pseudo- Impedance}

The process of residual deconvolution of the seismic wavelet from migrated volumes provides reflectivity volumes with an expanded frequency bandwidth. It has a long history of dev elopment in Petrobras. Internally known as iterative deconvolution, it was originally developed during the late 1970's by José Tassini and Emilson Evangelista, and subsequently improved by André Romanelli (Rosa, 2018). This process was subject to several refinements along the last two decades, with developments that include the stage of data preconditioning (Machado and Cunha, 2015), deconvolution process itself, and post-conditioning workf lows.

The pre-conditioning stage comprises attenuation of coherent and incoherent noises as well as residual phase correction of the wav elet. The phase correction is based on seismic/sy nthetic fitting at well locations as well as analy sis of some reference events such as the water bottom and
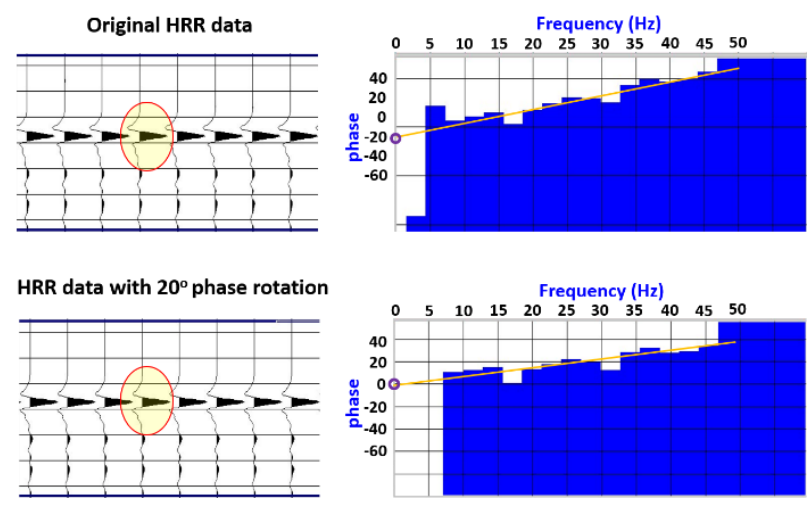

Figure 1: Estimating the residual phase correction. Top: HRR dat without phase rotation and their average phase spectrum Bottom. HRR data after $20^{\circ}$ phase rotation and their average phas $\epsilon$ spectrum The wavelet asymmetry observed in the original data is corrected after the phase rotation.

igneous intrusions.

Figure 1 illustrates the residual phase correction for the case of using the water bottom event as reference. By adjusting a linear trend to the relevant part of the phase spectrum, it is possible to determine the phase rotation that provides a null linear coefficient. In the particular case of this project, a phase rotation of 20 degrees was necessary. Subsequent correlation with synthetic traces at well locations validated this correction.

Af ter the residual wav elet deconv olution, it is necessary to simulate the spectral tendency of the subsurface reflectivity series. This is achieved by a spectral coloring shaping procedure (Rosa and Ulrych, 1991).

The spectrally shaped deconv olv ed data is then subject to time integration, which simulates a conversion from reflectivity to relative impedance or the so-called pseudoimpedance. The next stage inv olv es a process called HighResolution Pseudo-Impedance (PSIM), which consists of a combination of temporal and spatial structural filters that aim at the attenuation of the low-f requency noise amplif ied by the integration, while preserving the signal which is normally discharged in conventional filtering processes. Finally, the PSIM data is dif ferentiated with respect to time, resulting in a High-Resolution Ref lectiv ity (HRR) volume.

Figure 2 compares the (conventional) input data and the HRR data for 2-D window around Well-1. We verify the 
expressive increase in resolution, with continuous reflectors free from the usual artif acts amplified by typical processes of spectral enhancement, while preserving good correlation with the synthetic traces.

\section{Inversion of the high resolution data}

From 47 wells used in this project, 25 show a seismic-log correlation higher than $60 \%$ for the high-resolution data, considering a time window comprising the entire acoustic impedance log.

The time-depth calibration for broadband data is still more critical than for conventional data, since a small mismatch produces a significant decrease in the synthetic/seismic cross correlation due to the bandwidth expansion.

The usual tools provided by commercial inversion sof tware for the seismic wavelet estimation, which are based on least-squares minimization between seismic and sy nthetics, were not developed for broadband data. This method is efficient to estimate wav elets for conventional data, but inadequate for high-resolution data, such as HRR.

The size of the time window required for the wavelet estimation has also to be readdressed. For a satisf actory estimation of the amplitude spectrum, this size needs to be, at least, three times the size of the wavelet, especially to capture the amplitudes associated with the low frequencies. However, not only in this particular project, but in most standard projects, the sonic and density logs have a limited depth range, with an extent that may be inadequate ev en for conventional data. To cope with these issues, we opted for an alternative strategy for wavelet estimation, with a combination of automatic tools and manual adjustments.

The first step of the wav elet estimation is based purely on seismic data (without any well-related information). A large number of traces are used, with a larger time window than usually involved in conventional inversion, allowing for a

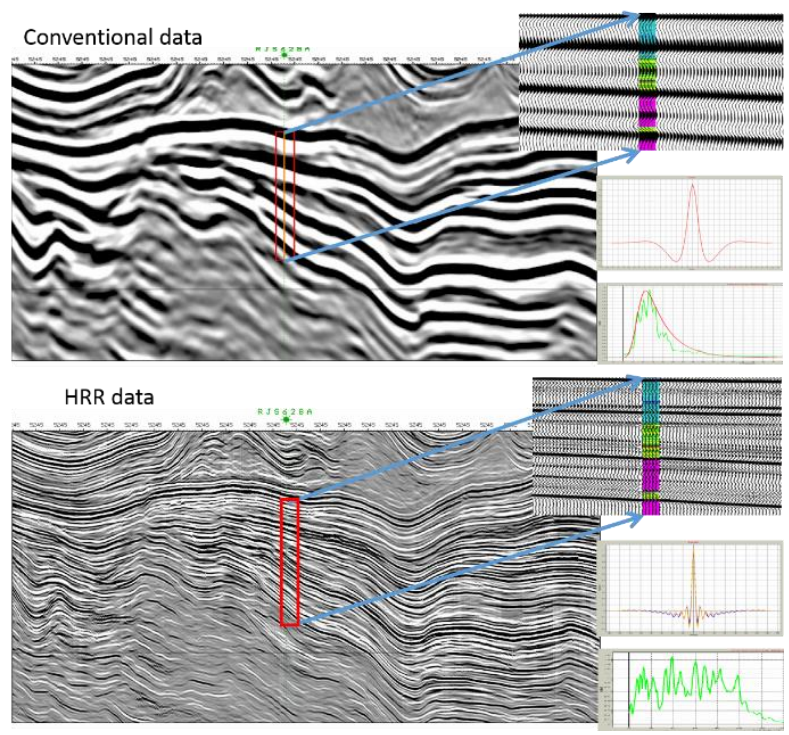

Figure 2: Top: Conventional data. Bottom HRR data. Amplifiec windows show good correlation of both with respective weli derived synthetics (each with their own wavelet). more robust estimation. Figure 3 compares the average seismic spectrum, the seismic-derived statistical wav elet spectrum, and a manually edited version of the wavelet spectrum to produce a smoother version. The next step comprises the least-squares adjustment of phase and amplitude, using the edited statistical wavelet as a priori information with a strong weight. With this approach, the least-squares adjustment is used only to guarantee that the seismic amplitudes honor the amplitude from the sy nthetics deriv ed from the well logs. The phase correction was neglectable and thus set to zero.

Figure 4 shows the final wavelets estimated for the conventional and the high resolution data, as well as their corresponding amplitude spectra.

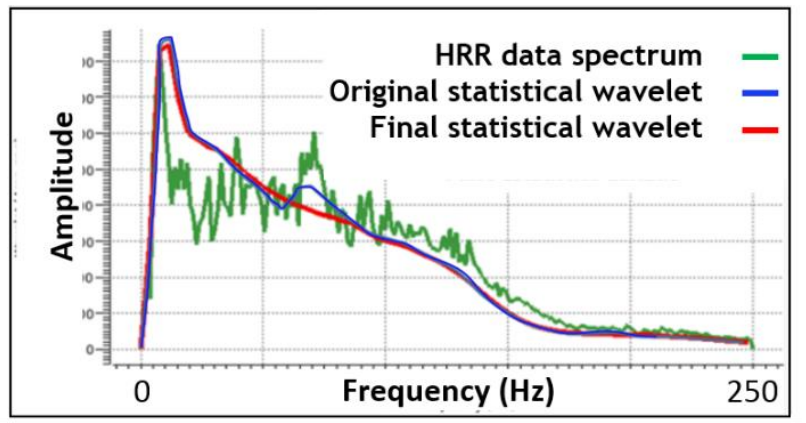

Figure 3: Comparing seismic spectrum (green) with originai statistical wavelet (blue) and its edited version (red).

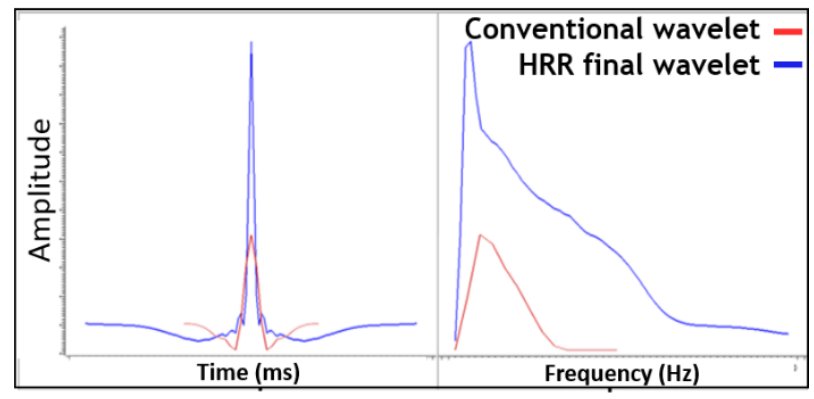

Figure 4: Superposition of final estimated wavelets for conventional data (red) and HRR data (blue). The HRR wavelet is broader, allowing for more low frequency content and with much smaller side lobes.

Regarding the inversion parametrization, since the highresolution data is, in many aspects, different from conv entional data, some considerations were necessary to serve as a guide for this project:

- Low-noise input data;

- More significant reflectors when compared to conv entional data;

- Reliable data down to $4 \mathrm{~Hz}$.

Lateral continuity constraints were used to provide smoother inversion results. Furthermore, we followed the two-step approach for the low-f requency insertion, which provides a more laterally continuous result, removing subvertical artif acts (Sobreira et al., 2014; internal report). We used merge frequencies of $4 \mathrm{~Hz}$ in the first step and $12 \mathrm{~Hz}$ in the second step, with the second-step model obtained from the output of the first-step inv ersion. 


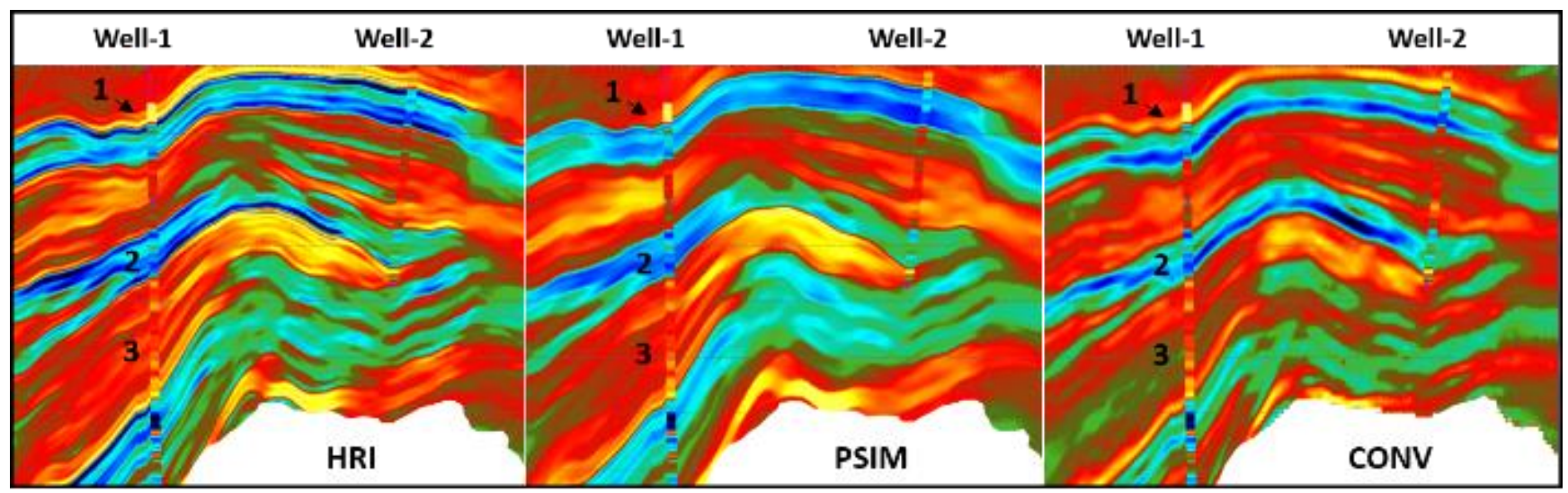

Figure 5: Results from three different approaches to acoustic inversion for a session crossing two wells. Hot colors (yellow to red) correspond to low impedances, while cold colors (light green to dark blue) correspond to high impedances. A trapezoidal band-pass filter (3.5Hz-5.5Hz$137.5 \mathrm{~Hz}-162.5 \mathrm{~Hz}$ ) was applied to the seismic traces as well as to the well logs. We verify that the HRI approach shows better correlation witr. the well logs when compared to the other two approaches.

\section{Analysis and quality control of results}

The results were analyzed in $1-D$, by comparing the inv erted traces with the logs at the well positions, and in 2$\mathrm{D}$, by comparing the seismic section along the wells. We also compare the acoustic impedance derived from the sonic and density logs with three versions of the inv ersion: conventional inversion (CONV), pseudo-impedance inversion (PSIM), deriv ed from the integration of the highresolution data (which has no low-f requency components), and the high-resolution inversion (HRI), which blends the interactiv e-deconv olution large bandwidth seismic and the sparse-spike seismic inv ersion. Finally we show the results of blind tests.

\section{Pseudo-wells and sessions}

To illustrate the concept that the high-resolution inversion produces superior results in terms of resolution and more reliable absolute values of acoustic impedances, we constructed Figure 5. It compares the results from three inversions: Conventional, PSIM, and HRI. For a session that crosses two wells (Well-1 and Well-2), the examination of this figure verifies that:

- The transitions between lay ers are smoother in the conventional inversion. The more abrupt contrast shown in the other two inversions is more geologically consistent with the discontinuous character of stratigraphic units.

- The $\mathrm{HRI}$ is capable to identify thin $(10 \mathrm{~m})$ anhy drite layers (dark blue in region 1), and, although it is present also in PSIM, its amplitude (tonality) shows a small relative contrast. We observe a similar behavior in other high relative impedance features (dark blue lay ers) in region 2 , as well, in region 3.

- The smaller (seismically related) content of low frequencies in the conventional inversion produces incorrect acoustic impedance (greenish colors) in thicker layers, such as the Piçarras Formation indicated in region $\mathbf{3}$, as attested by the well log.

- The dark blue feature in region 2 would be interpreted as a single body in the conventional inversion, but as three independent bodies (and more conform to local layer attitudes) in the HR inversion.

- In general, the HR inversion combines the stratigraphic details provided by the PSIM inversion with a more rigorous amplitude control provided by the sparse-spike inv ersion.

In order to complement these observations, Figure 6 compares impedance logs (full bandwidth up to $175 \mathrm{~Hz}$ ) of Well-1 and the traces from the HRI and CONV inversions. A visual inspection shows that (except for the last $50 \mathrm{~ms}$ ) the HRI inversion correlates better with the well log than the CONV inversion. The superiority holds in quantitative terms: 0.9 for $\mathrm{HRI}$ and 0.84 for CONV. It is worthwhile to point out that this result is even more surprising when we consider that the CONV inv ersion used the well inf ormation to a larger extent (up to $6 \mathrm{~Hz}$ ) than the $\mathrm{HRI}$ inv ersion (up to $4 \mathrm{~Hz}$ ) to construct the low-f requency model.

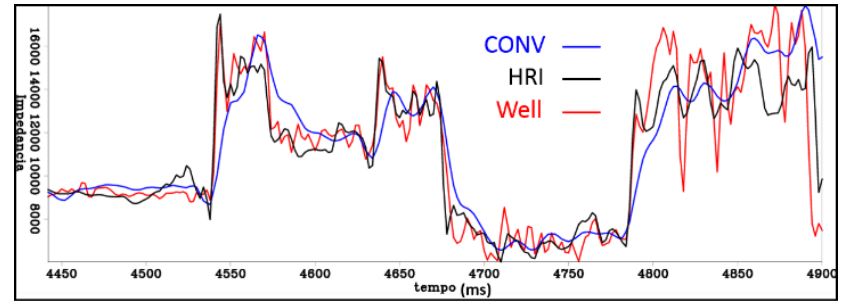

Figure 6: Acoustic Impedance logs: (red) from Well-1; (blue) from conventional inversion; (black) from high resolution inversion. The HRI result reproduces the sharp impedance contrasts present in the well log for a bandwidth up to $175 \mathrm{~Hz}$.

\section{Blind Tests}

In order to v alidate our conclusions, fiv e wells were chosen to be used as blind tests (not used in any part of the inversion workflow). We present, for each of the wells in table 1, two values of correlation with the seismic inversions at the well locations. Values on the left correspond to full bandwidth, and on the right (bold face) with a low cut of $6 \mathrm{~Hz}$. 


\begin{tabular}{|c|c|c|c|c|c|c|c|c|c|c|}
\hline Poço & \multicolumn{2}{|c|}{ Well-3 } & \multicolumn{2}{|c|}{ Well-4 } & \multicolumn{2}{|c|}{ Well-5 } & \multicolumn{2}{|c|}{ Well-6 } & \multicolumn{2}{|c|}{ Well-7 } \\
\hline CONV & 75 & $\mathbf{5 1}$ & $\mathbf{6 0}$ & $\mathbf{4 4}$ & $\mathbf{9 0}$ & $\mathbf{5 0}$ & $\mathbf{8 7}$ & $\mathbf{7 3}$ & $\mathbf{8 9}$ & $\mathbf{7 1}$ \\
\hline HRI & $\mathbf{7 9}$ & $\mathbf{6 3}$ & 60 & $\mathbf{4 5}$ & $\mathbf{9 5}$ & $\mathbf{7 2}$ & $\mathbf{7 9}$ & $\mathbf{8 0}$ & $\mathbf{9 1}$ & $\mathbf{8 0}$ \\
\hline
\end{tabular}

Table 1: Correlation values (in \%) between well log acoustic impedances and seismic inverted traces for the two approaches: CONV and HRI. Values on the left correspond to full bandwidth, and on the right (bold face) with a low cut of $6 \mathrm{~Hz}$.

Because the insertion of the low frequencies deriv ed from wells inv olv ed different merge f requencies, presenting the values with the $6 \mathrm{~Hz}$ low cut allows for a comparison in a bandwidth less susceptible to the influence of the well derived low frequency model.

For the full bandwidth results, correlations for the $\mathrm{HRI}$ are equal or superior compared to CONV in 4 of the 5 wells analy zed, while for limited bandwidth the HRI correlations are always superior. Notice that almost all correlations decrease when the low frequencies are absent. This result is expected even in the case of blind tests since this low frequency band contains inf ormation from neighbor wells. In the specif ic case of Well-4, both inversions present a relativ ely low correlation due to imaging deficiencies in the region where this well is located.

\section{Positioning an injector at a border of the field}

The distance between injectors and producers should be as large as possible in order to optimize the injected fluid pathways inside the reservoir, enhancing the oil production, and av oiding premature water breakthrough.

The best positions for injector wells are ty pically located in lower structures of reserv oirs to maintain the pressure and optimize the oil sweep by differential density and gravity. The lake bathy metry is a key control for facies deposition of pre-salt reservoirs (Faria et al, 2017). As consequence, the positioning of injector wells in lower structures increases the risk of drilling clay-bearing carbonate facies, penetrating low porosity and permeability rocks.

Therefore, an important part of the geological characterization of the pre-salt reservoirs is the prediction of the most probable regions where non-reservoir facies are present, and where respective transition zones, from clear to clay -bearing carbonates, occur in order to optimize the drainage.

Figure 7 presents some logs from a well located at a region characterized by the occurrence of transitional facies, near the west border of the field. The arrows in the figure indicate a zone of low porosity due to the presence of clay minerals in the carbonate rocks.

The results from the conventional and the high-resolution impedance inversions are presented in a session across this well (Figure 8). The gain of resolution achieved with the $\mathrm{HRI}$ approach signif icantly reduces the uncertainties to characterize the geometries of the two facies.

In addition to a better delineation of the carbonate lens rich in clay minerals the high resolution inversion has been ef $f$ ective in the delineation of lateral $f$ acies transition as well as the interdigitation of associated facies. The high porosity and permeability reserv oir zone (dark blue) as well as the thin clay-rich layer (light blue to green) are much better def ined, and with a more geologically consistent geometry in the $\mathrm{HRI}$ than in the CONV inversion. The gradual interdigitation between the two facies becomes ev ident.

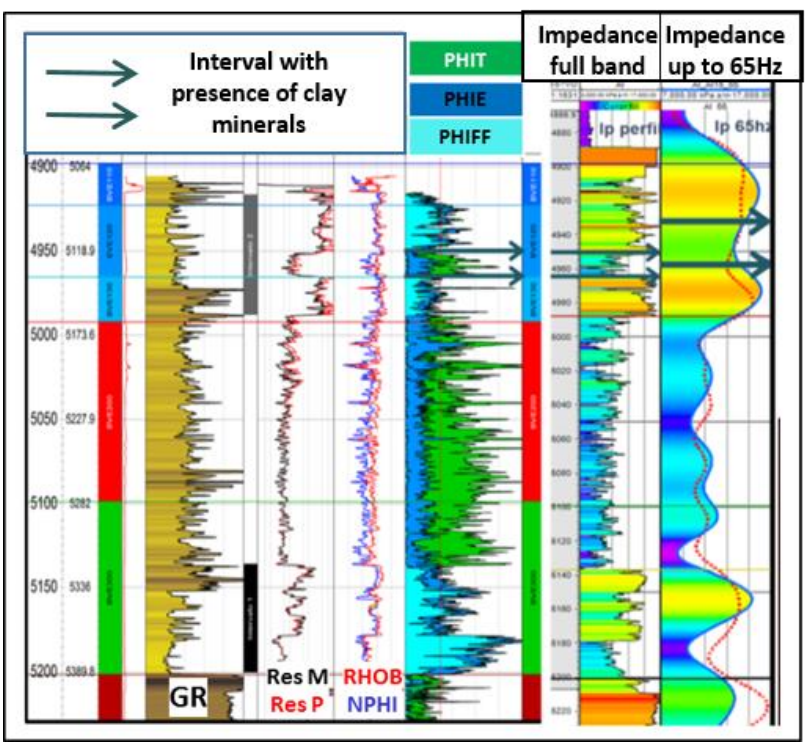

Figure 7: On the left several logs showing a layer with clay minerals indicated by the interval between the two arrows. On the right two versions of the well-based acoustic impedance, one in full bandwidth, and the other with a $65 \mathrm{~Hz}$ high cut filter. We verify that the limitation of the bandwidth decreases the resolution of the clayish layer not only in terms of impedance contrast (amplitude) but also in terms of thickness and positioning.

Four well locations for the new injector were considered at $f$ irst, based in integrated studies of flow simulation, risk analy sis for the presence of clay minerals at the reserv oir and return on investments. Flow simulations fav ored two locations, while geological risk analy sis recommended the other two locations. It became critical to perf orm a detailed study using the high-resolution data, to reduce the geological uncertainty and to support a saf e location suited for the drainage optimization.

The well was drilled and the seismic prediction of highquality reserv oir rocks proved to be suitable for the fluid injection requirements.

\section{Final Comments and Conclusions}

We presented in this paper the general workflow for producing high resolution reflectivity (HRR) volumes. We also discussed the required adaptations in the conventional inversion workflow in order to cope with the particularities of the HRR data.

To evaluate the results we compared three inversion approaches: conventional inversion, pseudo-impedance inversion and high-resolution inv ersion. The adv antages of this new methodology are quite representative, as demonstrated by the sessions across some wells. The improvement in resolution is testif ied by the quality control results, and supported by well data. Blind tests also attest the capability of $\mathrm{HRI}$ to capture high frequency inf ormation from seismic. 
The use of the HRI volume supported the location of new injectors and producers in the field. This approach to inversion brings significant gains not only in the characterization of the reservoir internal geometries (and associated depositional system), as well as in quantitative estimation of reservoir properties with resolution more compatible with flow simulation models.

\section{Acknowledgments}

We would like to thank Petrobras for the opportunity to produce and present this work. We acknowledge also the contribution of Andre Romanelli Rosa and Guenther Schwedersky for helping to forge many of the ideas presented in this work.

\section{References}

Av seth, P., T. Mukerji, and G. Mavko, 2005, Quantitative seismic interpretation: Applying rock physics tools to reduce interpreta-tion risk: Cambridge University Press, https://doi.org/10.1017/ CBO9780511600074.

Cunha, C. A., Ruthner, M. P., Gontijo, R. C., e Almeida, S. H. M. 2013. Statistical Seismic Facies Estimation from Pseudo Impedance Data. 75th Conf erence \& Exhibition of the European Association of Geoscientists and Engineers, doi: $10.1190 /$ sbgf 2013-131

Faria, D. L., Reis, A. T., Souza Jr., O. G., Threedimensional stratigraphic-sedimentological forward modeling of an Aptian carbonate reservoir deposited during the sag stage in the Santos Basin, Brazil. Marine and Petroleum Geology, 88, 676-695, doi: 10.1016/j.marpetgeo.2017.09.013

Latimer, R.B., 2011. Inversion and interpretation of impedance data, in: Interpretation of Three-Dimensional Seismic Data. Society of Exploration Geophy sicists and American Association of Petroleum Geologists, pp. 309350. doi:10.1190/1.9781560802884.ch9.

Machado, M., and Cunha, C., 2016. Structure-oriented Filter by Domain Decomposition: 14th International Congress of the Brazilian Geophy sical Society, 1285-1290, doi: $10.1190 /$ sbgf 2015-255

Rosa, A. R., and Ulry ch, T., 1991, Processing via spectral modeling: Geophy sics, 56, no. 8, 1244-1251, doi: $0.1190 / 1.1443144$

Rosa, A. L. R., 2018. The Seismic Signal and its Meaning: edited by the Society of Exploration Geophy sicists (SEG), doi: $10.1190 / 1.9781560803348 . \mathrm{fm}$

Vernik, L. Seismic petrophysics in quantitative interpretation. edited by the Society of Exploration Geophy sicists (SEG), doi: 10.1190/1.9781560803256.f m

Teixeira, L., Cruz, N., Silvany, P., Fonseca, J. 2017. Quantitative Seismic Interpretation integrated with well-test analysis in turbidite and presalt reservoirs: The Leading Edge, November, Vol 36, No 11, pp 931-937, doi: 10.1190/tle36110931.1
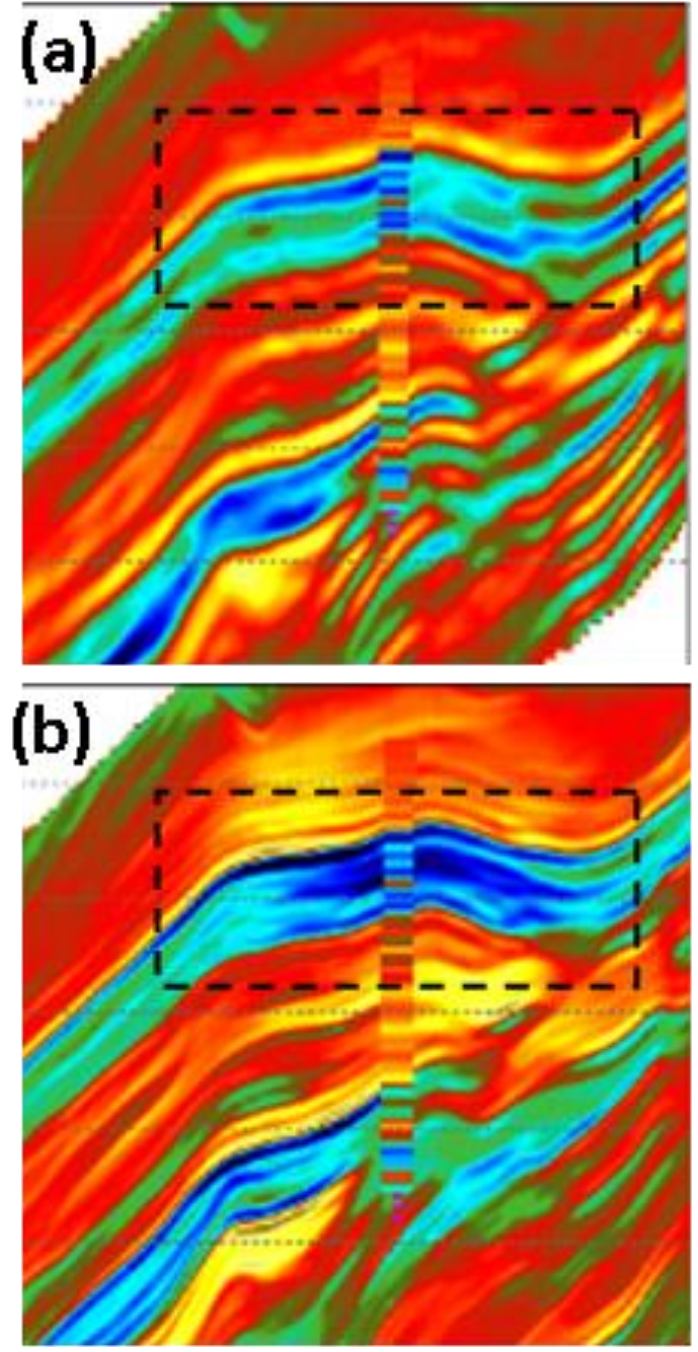

Figure 8: (a) Conventional inversion (CONV) (b) Higr. resolution inversion (HRI). 